\title{
Wireless Body Sensor Design for Intra-Vaginal Temperature Monitoring
}

\author{
João F. R. Garcia \\ Instituto de Telecomunicações, \\ University of Beira Interior \\ Covilhã, Portugal \\ jgarcia@it.ubi.pt
}

\author{
João M. L. P. Caldeira \\ Instituto de Telecomunicações, \\ University of Beira Interior \\ Covilhã, Portugal \\ Pol. Inst. Castelo Branco, Portugal \\ jcaldeira@it.ubi.pt
}

\author{
Joel J. P. C. Rodrigues \\ Instituto de Telecomunicações, \\ University of Beira Interior \\ Covilhã, Portugal \\ joeljr@ieee.org
}

\begin{abstract}
Sensor nodes are small devices able to collect and retrieve sensorial data. The use of these sensors for medical purposes offers valuable contributions to improve patients' healthcare, both for diagnosis and therapeutics monitoring. An important and common parameter used on healthcare diagnosis is the body temperature. It is monitored on several matters related with gynecological and obstetrics issues but, usually it is measure at the skin surface. Then, this paper proposes the design concepts of a new intra-body sensor for long-term intra-vaginal temperature collection. The embedded IEEE 802.15.4 communication module allows the integration of this sensor in wireless sensor networks for remote data access and monitoring. It is presented the sensor architecture, the construction of the corresponding testbed, and its performance evaluation. This sensor may be used on several applications, including fertile and ovulation period detection, and preterm labor prevention.
\end{abstract}

\section{Categories and Subject Descriptors}

D.3.3 [Systems and Technology]: System design issues, and performance modeling

\section{General Terms}

Measurement, Design

\section{Keywords}

Body sensor networks, wireless biosensor, temperature monitoring and e-Health

\section{INTRODUCTION}

Sensor networks evolved with a lot of expectations in terms of research due to the large applicability and possibility for development, especially in the wireless sensor networks (WSNs).

Permission to make digital or hard copies of all or part of this work for personal or classroom use is granted without fee provided that copies are not made or distributed for profit or commercial advantage and that copies bear this notice and the full citation on the first page. To copy otherwise, to republish, to post on servers or to redistribute to lists, requires prior specific permission and/or a fee. BodyNets '10, September 10-12, Corfu Island, Greece

Copyright (C) 2010 ACM 978-1-4503-0029-2/10/09... \$10.00
Low power consumption and low data rates are the most important features for WSN applications [1]. Nowadays, due to the technological evolution of e-health one can have sensors for all sizes and with lots of features, sensors that can even be placed inside (intra-body sensor) or outside (inter-body sensor) the human body, which are in contact with skin [2]. This evolution drives to new proposals that try to be easier to use, more comfortable and miniaturized as much as possible. All these types of sensors deals with many constrains on resources such as energy, memory, computational speed, and bandwidth. Furthermore, with this evolution those platforms are very helpful for medical diagnoses and treatment methods because some of them can only be recorded by electronic sensors. All the studies carried out over the past years predict that different parts of the body have different skin temperatures and can easily be affected by external factors [3]. The increase in body temperature is the first indicator of an inflammation, fever, or physical injuries.

The monitoring of this female physiological parameter may help in understanding and characterizing some of the known fertility issues [4]. Medical research devoted to the understanding of these fertility issues concluded that the core body temperature could be used to detect and identify some symptomatic female stages, such as ovulation and fertile periods [5].

Given the lack of available proposals to collect long-term core body temperature, this paper proposes the architecture to construct an intra-body sensor for acquisition and monitoring of core body temperature. The proposal is based on a wearable device to be placed inside female cervix. Size of the sensor platform, comfort for women, and wireless communications were some of the requirements taken into account in the conceptual design of this new sensor. The measurement and control of intra-vaginal temperature is used to inform woman when her fertile period occurs, for example. This period is referred in the literature as the time with best probability of getting pregnant [6]. In a careful search and to the best of our knowledge, we conclude that besides the existing motes our proposal has newest features with major importance for woman. Most of the treatments that need these female's parameter control are performed by manual measurements of body temperature with thermometers and respective registration of these measures on a paper chart [4].

Besides this application, the proposed sensor device aims to achieve other objectives. It may help on preterm-labor prevention, effectiveness of gynecological therapeutics, and support new contraception methods discovering. This paper is the result of a work in progress and shows the evolution of a challenging project 
on e-health, since [7] and [8] proof that this project is for sure a great contribution for e-health technology.

The remainder of this paper is organized as follows. Section 2 reviews available devices and gathers contributions to this proposal. Section 3 describes the essential requirements that will be featured in the new sensor device while the sensor prototype design is explained in Section 4. Section 5 focuses on the results achieved with a testbed and, finally, Section 6 concludes the paper and presents further research directions.

\section{RELATED WORK}

Biosensors has several applications and given the available technological development many motes were created, performing many physiological parameters monitoring [2]. This section deals to some of the recent proposed motes and applications, most of them, focusing on monitoring and analysis of human body temperature.

Several bio-motes such as Shimmer [9], Eco [10], MICAz [11], SAND [12], and DuoFertitlity [13] have been developed and presented. Most of them measure health parameters like temperature, blood pressure, EMG (electromyography), ECG (Electrocardiography), EEC (electro and motion) as shown on Figure 1.

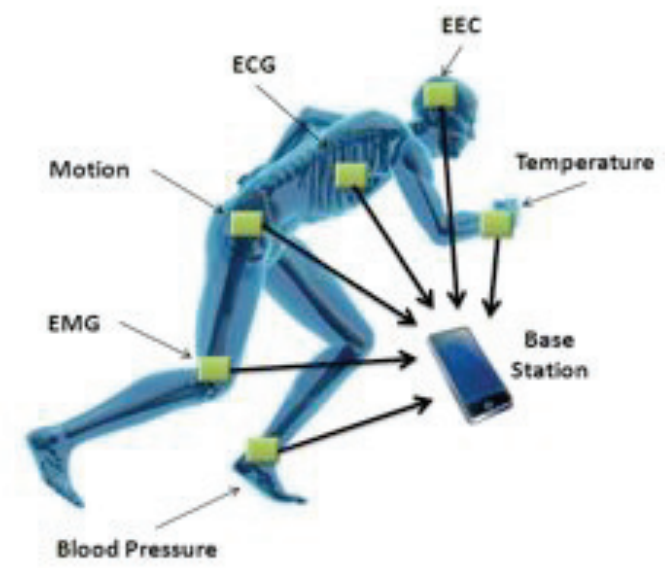

Figure 1. Illustration of a typical body sensor network.

First and most important to our project is the Shimmer (Sensing Health with Intelligence, Modularity, Mobility and Experimental Reusability), which is a powerful small wireless sensor platform. It uses a TinyOS operating system. Some features of this platform include the following: wireless communication (Bluetooth and IEEE 802.15.4), microSD (micro Secure Digital) card slot allowing up to 2 Gigabytes of data, embedded 3-axis accelerometer, and a Texas Instruments (TI) MSP430 microcontroller. It uses a $250 \mathrm{mAh}$ rechargeable battery for power supply. This sensor fits on a Printed Circuit Board (PCB) with $44 \mathrm{~mm} \times 20 \mathrm{~mm} \times 21 \mathrm{~mm}$. This platform has a lot of potential for health applications due to its communication technologies and technical features [9]. According to our expectations and needed features, this platform exceeds them in terms of size, shape, and technical features (Bluetooth, 3 axis-accelerometer, and interface connections).

The Eco mote is a miniaturized sensor mote with a dimension of $13 \mathrm{~mm} \times 10 \mathrm{~mm} \times 8 \mathrm{~mm}$. It uses a nRF24E1 module that is a Radio Frequency (RF) transceiver with an embedded 8051 microcontroller. It has a 3-axial acceleration sensor, a temperature sensor, and a light sensor. For powering, this sensor uses a $30 \mathrm{mAh}$ rechargeable battery [10]. Owing to its small sensor size, it has limitations when collecting long-term data. No extra memory support (like microSD slot) is available on this sensor; so long applications cannot be supported. This mote was used to detect spontaneous motion monitoring of preterm infants and in an interactive art performance. This mote allows collection of wide sensing parameters from multiple types of devices. This mote fits almost all of the features that we need but fails in the most important factor for long-term data applications, the memory card support.

The MICAz is a tiny wireless measurement system mote from Crossbow. It provides 2.4 GHz IEEE 802.15.4 compliant RF transceiver designed specifically for deeply embedded sensor networks with low-power consumption. This mote uses an ATmega128L, which is a low-power microcontroller and has a 51-pin expansion connector that supports additional analog inputs, digital I/O, I2C, SPI, and UART interfaces. All these interfaces make it easy to connect to a wide variety of external sensor boards, such as expansion connector for light, temperature, RH, barometric, acceleration/seismic, acoustic, magnetic, among others. Similar to TelosB, this mote uses 2 x AA powering batteries. Like Eco this mote does not have memory slot support and exceeds in size and shape according to our application [11].

The Small Autonomous Network Device (SAND) is a modular application development platform for small wireless sensor or actuators. This platform follows a typical mote design with a small PCB and an embedded microcontroller, many interfaces, and a wireless communication block (IEEE 802.15.4). This platform has the possibility of adding new blocks measuring $14 \mathrm{~mm}$ of diameter and a volume of $1.5 \mathrm{~cm}^{3}$ each, and with a different functionality. Due to all its features and size this mote is well used for healthcare patients monitoring systems. SAND motes are easily assembled and C-programmable small sized enabling fast and reduced cost field testing. As a result of its cylindrical shape, a CR1225 coin cell battery is used to provide power on system [12]. In contrast with our proposed system this mote is not a good option due to the system design applied to connect each different module. Each module was designed for a specific feature or operation and this mote does not have an extra memory slot to allow constant data record.

Finally, DuoFertility project created a system to predict women's fertile period by measuring and controlling the skin's temperature. This system comprises two modules, a wireless sensor mote placed under the arm of the monitored women and a remote reader unit. The sensor mote, used all the time, collects measurements of skin temperature and the reader unit could retrieve these measures on demand. This unit indicates with light scale the probability of the fertile period occurring. In this proposal, we can see that skin temperature changes with the environment where it is taken. Then, getting core body temperature leads to more reliable results. 
Taking into account other available motes solutions, we can easily conclude that some of them do not fit the physical and electrical features required, like TelosB [14] and Tyndall [15], which is the reason why they are not described. Next section will focus on system requirements.

\section{SYSTEM REQUIREMENTS}

This section studies the system requirements that lead to a proposal of the sensor architecture in terms of its anatomic shape and size to be placed inside a vagina. The interaction with the sensor should be wireless and the sensor should have the ability to operate in a stand-alone mode.

Due to the working place of the proposed sensor, it has to respect some anatomic limitations, namely, it should be comfortable and easy to place inside the vagina. Following a medical recommendation the sensor has to fit in a container with about $60 \mathrm{~mm} \times 15 \mathrm{~mm}$ of area. Therefore, a size of $30 \mathrm{~mm} \times 13 \mathrm{~mm}$ was the target for the main board with a microcontroller, a micro SD card slot, a transceiver (IEEE 802.15.4), and a computer interface included. The use of a shape similar to a trivial tampon seems to be a perfect choice. It is well known by women, easy to use, anatomically perfect, and it has the size to accommodate all the above-mentioned features. The tampon like the shape presented in Figure 2 has a conceptual design of the new proposed sensor. As may be seen, the thermistor is placed on the top of the container, the electronic circuit in the body of the container, and the battery on the tail.

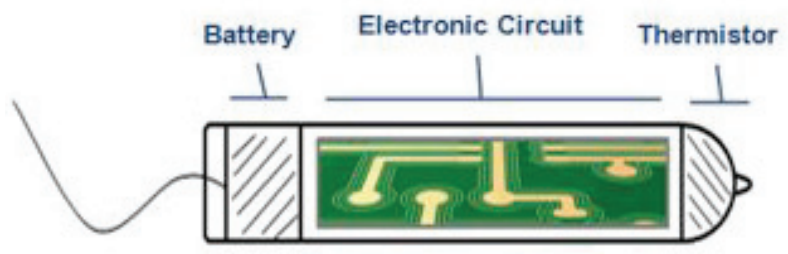

Figure 2. Conceptual design of the new intra-vaginal temperature monitoring sensor mote.

With woman's comfort in mind, the sensor should be operated when placed inside vagina. This feature avoids the need to take it out every time it is necessary to perform an operation to pick up the collection of measured temperature values. This feature will belong to this new sensor platform with the inclusion of a wireless communication module supporting the standard IEEE 802.15.4. This feature also allows re-configurations to the sensor operation mode without a physical connection to the programming dock/station.

Like many other nodes, a small rechargeable battery is considered. This battery supports a long lifetime, also due to the low-power system consumption. The power consumption is the main feature for any wireless sensor node in a way to achieve long-term operation. In WSNs is expected that all nodes have a transceiver layer and a battery to get mobility, and spend most of the time in low-power state, waking up only when readings and transmissions are required [2].
The sensor is designed for collecting intra-body temperature measures for long periods of time (e.g. during a menstrual cycle). Thus, this feature will be guaranteed with the inclusion of a microSD slot for a large amount of data collection. The power supply must also provide long periods of operation time. The sensor design includes a rechargeable battery with regular voltage of $3.6 \mathrm{~V}$ and capacity of $450 \mathrm{mAh}$, which can easily cover the required power of all components for long periods of time operation.

For temperature measurements it was included a thermistor. This thermistor will be used to measure the intra-vaginal temperature.

\section{TESTBED}

The architecture design proposed in this paper follows the abovementioned requirements and the proposed node includes the technical features to address these requirements. This sensor node requires a small size and needs a long lifetime in order to satisfy a large number of applications [1]. The sensor node includes a small PCB with a microcontroller, a small rechargeable battery, an external memory card, and a low power radio chip. Figure 3 presents the blocks diagram of the architecture used in the construction of the proposed sensor testbed.

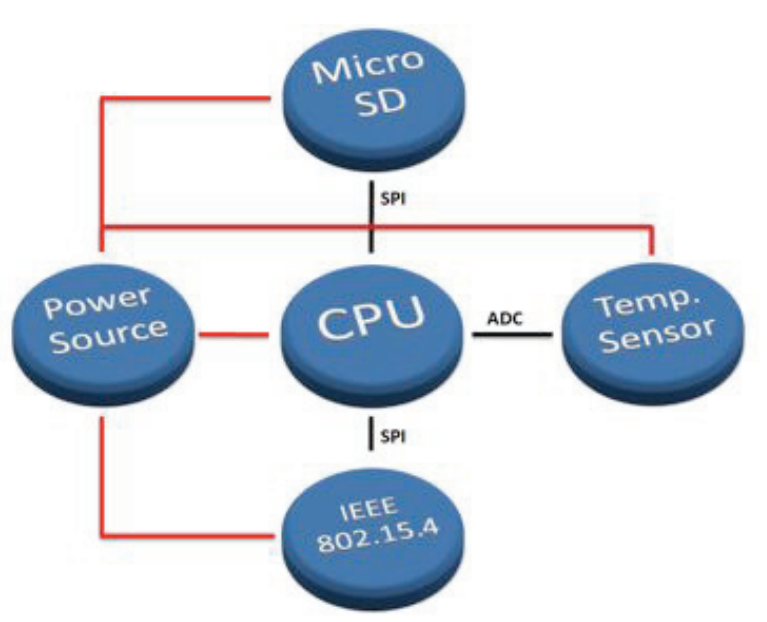

Figure 3. Sensor's block diagram of the proposal.

Following, it is presented a detailed description of identified and selected components to create the sensor. It includes the following elements: microcontroller (CPU), power source, data storage, communication device, and the sensor to collect temperature values.

The microcontroller is the main element of the sensor because it influences the rest of the solution; a microcontroller with low cost and low dynamic power consumption is essential. Then, the Texas Instruments MSP430F1xxx series [16] was the choice. Other motes use the same microcontroller family, such as Telos [14] motes. This microcontroller has fast start-up times, being very important in low duty cycle embedded applications. For these reasons, the selection of the MSP430F1611 is appropriated taking 
into account the following characteristics: 10Kbyte RAM, $48 \mathrm{Kbyte}$ Flash, supports up to $8 \mathrm{MHz}$, and offers $1.8-3.6 \mathrm{~V}$ operation which guarantee good expectations for all sensor development. Furthermore, this micro was chosen because the highest features presented allowing the possibility of the integration of new future sensors for other applications.

For communication purposes, the Chipcon CC2420 [17] is a true single-chip with 2.4GHz IEEE 802.15.4 compliant RF transceiver, perfect for low power and low voltage wireless applications. This chip has a digital direct sequence spread spectrum baseband modem providing a gain of $9 \mathrm{~dB}$ and an effective data rate of 250 kbps. The antenna adopted seems to be a good option because it is intended for $2.4 \mathrm{GHz}$ applications using Zigbee ${ }^{\circledR}$ [18]. This antenna is easy to integrate, has a high efficiency, and is light weight. In order to obtain high efficiency, the antenna was designed for use with no ground beneath the antenna but this ground plane must not extend underneath the antenna itself. The size of the antenna is $6.1 \mathrm{~mm} \times 3.9 \mathrm{~mm} \times 1.1 \mathrm{~mm}$, and with a weight of $0.05 \mathrm{~g}$.

For a sensor node, in many applications and usage, it is highly relevant to have big data storage. Then, the memory size is a limitation because the microcontroller only has 10kbyte RAM and $48 \mathrm{Kbyte}$ Flash, which is not enough to record continuous data. The major advantage of the sensor is providing an external microSD card with up to 2 Gbytes of memory. This feature adds a way to use applications where the storage amount of data is a real problem, mainly, in most cases where the small size of the motes is a key issue.

In this proposed testbed, as shown in Figure 4, a power supply is used to power the testbed but, for a final prototype, Lithium batteries already tested will be used. The chosen battery is a Lithium battery from GMBPower [19], model ER13150 measuring $15,5 \mathrm{~mm} \times 13,5 \mathrm{~mm}$, with a capacity of $450 \mathrm{mAh}$. This battery was chosen particularly due its circular shape and large capacity. As a regular battery, the nominal voltage will decrease as the battery is discharged. In order to provide a regular voltage of $3.6 \mathrm{~V}$ on the system, a voltage regulator is needed.

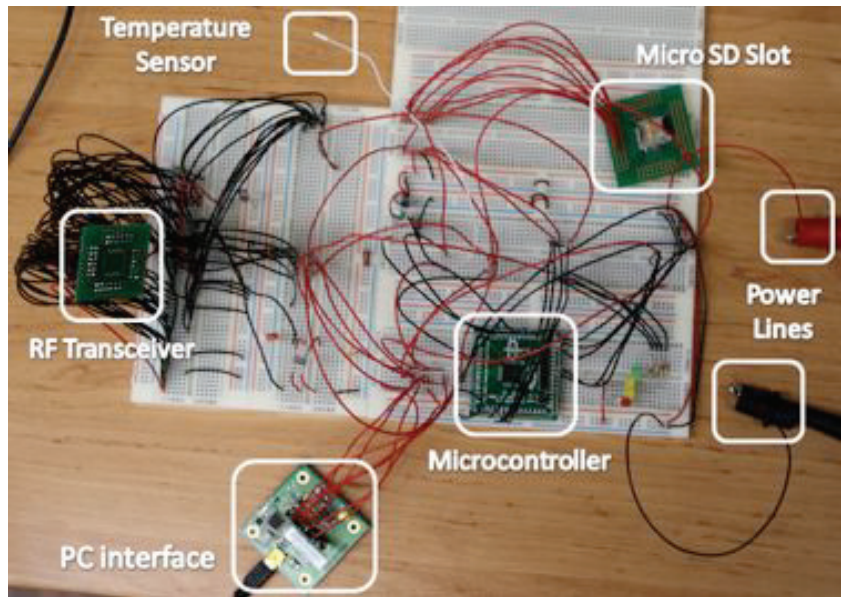

Figure 4. Physical testbed for Temperature Monitoring.

For the design of the temperature sensor, the MA100 [20] thermistor was chosen. It is a NTC Type MA Biomedical Chip thermistor developed by GE Industrial Sensing and exclusively used for biomedical applications. Its main features fulfill the requirements of our solution. Its sensitivity ranges from $0^{\circ} \mathrm{C}$ to $50^{\circ} \mathrm{C}$, with size of $0.762 \times 9.52 \mathrm{~mm}$. The size, shape, temperature ranges, and its approval for medical applications was the major reasons for this choose. To get more accurate temperature readings and taking into account its goal, the temperature sensor must be placed inside the female cervix, which is an ideal thermal source to reach the core body temperature. For this proposed architecture, the MA100 is embedded in the mote platform.

Using Zigbee [18] communication, temperature monitoring can be performed in a real-time mode. Besides, the sensor platform saves measured values in the embedded microSD card and it also sends them to external devices in Zigbee range area. This wireless communication is also used to transfer all the data stored in the sensor's microSD card on demand.

Another important feature of this solution is mobility. After mote activation and correct placement inside vagina, the woman can move freely and do whatever she wants with comfort. After switching on the data collection (operation performed through remote commands), the sensor starts measuring and continuously storing data in the microSD card. The monitored woman only has to take the sensor out when the date advised by the doctor has been achieved. During a long monitoring period, the sensor need only be removed for battery recharge.

For a regular medical observation, a doctor can use the mote to measure current core temperature of a patient in his office with a real-time connection to the sensor, as above described. On the other hand, he can connect to the sensor directly and retrieve all collected data to his computer. In both cases the doctor can monitor and control the evolution of this biological parameter observing a graphical representation of the measured values.

\section{PERFORMANCE EVALUATION AND VALIDATION}

As shown in Section 4, the temperature sensor was used to evaluate our sensor design. Supported by the computer application presented in [21] the temperature values were collected from natural environment and from a glass of water.

Several measures were performed to make sure the sensor was getting the temperature values correctly or if any temperature variations were registered. To validate the sensor performance, the same measures were performed with a digital multi-function Fluke 289 coupled with an $80 \mathrm{BK}-\mathrm{A}$ thermocouple probe as shown on Figure 5.

The tests were performed in permanent contact with water due to the real environment application of the body-sensor (inside vagina). These tests allow to simulating a humidity field like vagina. In real time, it was possible to check the collected values performed by the testbed with the temperature calibrator measured by the Fluke 289, as Figure 6 shows in detail. According to the collected values we can conclude that the testbed was calibrated and measure the real environment temperature. 


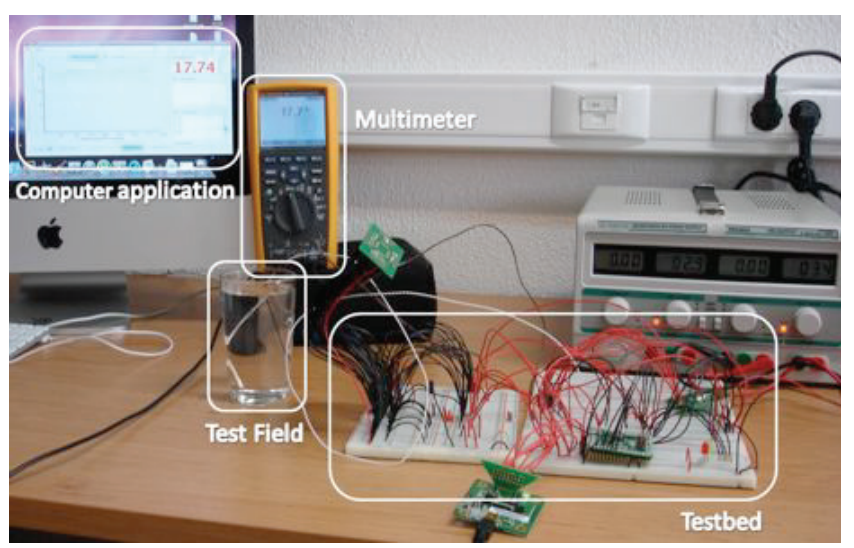

Figure 5. Experimental testbed validation for temperature measurements.

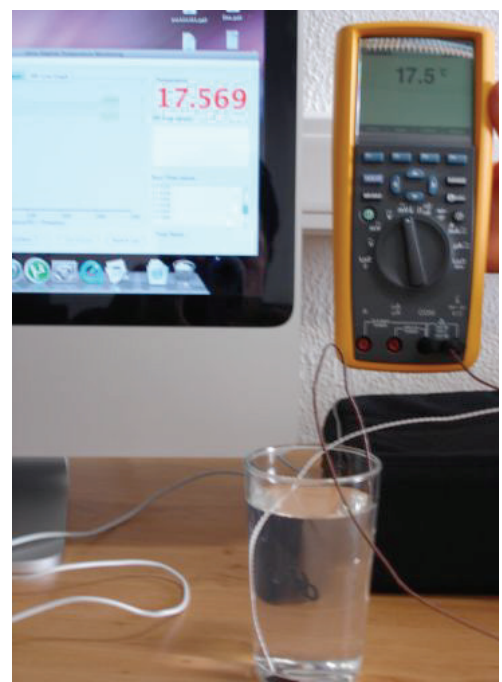

Figure 6. Detail of the comparison between temperature values collected by testbed and the temperature calibrator.

\section{CONCLUSIONS AND FUTURE WORK}

This paper presented the architecture design and the construction of a testbed of a new wireless body sensor for temperature monitoring. The proposed biosensor aims to collect female corebody temperature. To reach this objective, the sensor should be placed inside the vagina close to cervix. Therefore, the construction of this new body sensor has to take into account some constraints. An important restriction is the size, so the sensor dimensions cannot exceed $60 \mathrm{~mm} \times 13 \mathrm{~mm}$. Then, the enclosure of the new sensor was carefully planned with comfort in mind, and a tampon like shape was considered. The sensor should communicate wirelessly. To perform it, an IEEE 802.15.4 communication module was included in the architecture design and therefore, in the testbed. Another important feature is related to its ability to store long-term temperature measures. The proposed system includes a microSD card slot to store large amounts of data. The sensor should work for long periods of time in stand-alone mode. So, the use of a rechargeable battery was also considered. In the future, women could use this sensor for monitoring their core-body temperature. The control of this parameter helps them to detect their fertile and ovulation periods for fertility or contraceptive purposes. Other medical applications can be assigned to this sensor in studding possible relationships with variations in this parameter and contractions in pregnancy, preterm-labor prevention, effectiveness of gynecological therapeutics and support to discover new contraception methods.

The construction of this new biosensor belongs to our interests for future work. After, the system composed by the sensor and the corresponding computer application to communicate with it will be evaluated and validated by medical staff before being available for use.

\section{ACKNOWLEDGMENTS}

The authors would like to thank Prof. José Moutinho (M.D.), from Health Sciences Faculty, University of Beira Interior, Portugal, for his expertise and invaluable assistance in this project. Part of this work has been supported by the Instituto de Telecomunicações, Next Generation Networks and Applications Group (NetGNA), Portugal, in the framework of Project BodySens, and by the Euro-NF Network of Excellence of the Seventh Framework Programme of EU, in the framework of the Specific Joint Research Project PADU.

\section{REFERENCES}

[1] I. Khemapech, I. Duncan, and A. Miller, "A Survey of Wireless Sensor Networks Technology," in Proceedings of the 6th Annual PostGraduate Symposium on the Convergence of Telecommunications, Networking \& Broadcasting (PGNET), Liverpool, UK, 2005.

[2] A. Pantelopoulos, and N. Bourbakis, "A survey on wearable biosensor systems for health monitoring," in 30th Annual International Conference of the IEEE Engineering in Medicine and Biology Society (EMBS 2008), Vancouver, British Columbia, Canada, pp. 4887-4890.

[3] I. Campbella, "Body temperature and its regulation," Anaesthesia \& Intensive Care Medicine, vol. 9, no. 6, pp. 259-263, 2008.

[4] J. Beaudoin, and R. Marrocco, "Attentional validity effect across the human menstrual cycle varies with basal temperature changes," Behavioural Brain Research, vol. 158, no. 1, pp. 23-29, 2005.

[5] L. Ngalamou, and D. Rose, "Fertility information appliance," in Proceedings of the 15th IEEE Symposium on ComputerBased Medical Systems (CBMS 2002), Maribor, Slovenia, 2002.

[6] J. Meylan. "Diagnostic Methods in Female Infertility," November 2009; http://www.gfmer.ch/Books/Reproductive health/Diagnostic methods female infertility.html. 
[7] P. Neves, J. Caldeira, A. Mendes et al., "A Biosensor and Data Presentation Solution for Body Sensor Networks," in 7th Conference on Telecommunications (CONFTELE 2009), Santa Maria da Feira, Portugal, pp. 173-176.

[8] J. J. P. C. Rodrigues, J. Caldeira, and B. Vaidya, "A Novel Intra-body Sensor for Vaginal Temperature Monitoring," Sensors Journal, MDPI, Basel, Switzerland, vol. 9, no. 4, pp. 2797-2808, 2009

[9] B. Kuris, and T. Dishongh, "SHIMMER Hardware Guide REV 1.3," Intel Corporation, [October 2009, 2006].

[10] C. Park, J. Liu, and P. H. Chou, "Eco: an ultra-compact lowpower wireless sensor node for real-time motion monitoring," in Fourth International Symposium on Information Processing in Sensor Networks (IPSN 2005), UCLA, Los Angeles, California, USA, pp. 398-403.

[11] "MICAz datasheet - Crossbow," March 2010 http://www.xbow.com/Products/Product pdf files/Wireless pdf/MICAz Datasheet.pdf.

[12] M. Ouwerkerk, F. Pasveer, and N. Engin, "SAND: a modular application development platform for miniature wireless sensors," in International Workshop on Wearable and Implantable Body Sensor Networks (BSN 2006), Cambridge, MA, USA, pp. 170-175.

[13] "DuoFertility Nebpage," March 2010; http://www.duofertility.com
[14] J. Polastre, R. Szewczyk, and D. Culler, "Telos: enabling ultra-low power wireless research," in Fourth International Symposium on Information Processing in Sensor Networks (IPSN 2005), UCLA, Los Angeles, California, USA, pp. 364369.

[15] J. Barton, B. O'Flynn, S. Bellis et al., "A miniaturised modular platform for wireless sensor networks," in Proceedings of the 2005 European Conference on Circuit Theory and Design, Cork, Ireland, 2005, pp. 35-38.

[16] "Texas Instruments - MSP4301xxx," March 2010; http://focus.ti.com/paramsearch/docs/parametricsearch.tsp?fa milyId=911\&sectionId=95\&tabId=1527\& family=mcu.

[17] "Texas Instruments/Chipcon - CC2420," March 2010; http://focus.ti.com/docs/prod/folders/print/cc2420.html.

[18] "Zigbee Aliance webpage," March 2010; http://www.zigbee.org/.

[19] "GMBPower Battery datasheet," March 2010; http://www.gmbattery.com.

[20] "MA100 Biomedical Chip Thermistor," March 2010; http://www.gesensing.com/products/MA100.htm.

[21] J. M. L. P. Caldeira, J. A. F. Moutinho, B. Vaidya et al., "Intra-Body Temperature Monitoring using a Biofeedback Solution," in The Second International Conference on eHealth, Telemedicine, and Social Medicine (eTELEMED 2010), St. Maarten, Netherlands Antilles, 2010. 